\section{ENDOCRINE MAN}

\section{(A Study in the Surgery of Sex)}

By L. R. Broster, O.B.E., D.M., M.Ch., F.R.C.S. Wm. Heinemann, London. 1944. Price I2s. 6d.

The advance in research into the functions of the endocrine glands has opened up such a vast field of biological knowledge and speculation that any book which attempts to find a proper place for endocrinology in the biological approach to the study of life is bound to be welcome. It is doubtful if any one reviewer has the knowledge to assay the degree of success that the author has achieved in the wider aspects of his presentation, and this review is therefore more interested in the endocrine aspects to which the author has made notable contributions, namely in the surgery of the adrenal glands. The author's study of hermaphroditism and sex reversal in fish and fowl are interesting and illuminating, and it is not easy to realise that " in 1478 a cock was sentenced and burned to death at the stake in Basle for the heinous and unnatural crime of laying an egg." On p. 67 there is a reference to the anti-hormones of Collip and a supposition that they exist normally in the body, whereas it is now generally held that they are more probably anti-hormonic substances, generated when hormones obtained from the gland of a different species are injected.

In an introduction to a study of the individual endocrine glands, the author claims that his remarks will not be detailed or advanced, but "a kaleidoscope picture of impressions gathered in the pursuit of practical clinical application." This modest approach must temper a critical review, but unfortunate errors have crept in, as p. 72- "Posterior lobe extracts are said to cause hypertrophy of the interstitial tissue of the testes," and p. 78 " Recent work on testicular tumours has shown that some forms actually secrete hormonal substances such as prolactin." Nevertheless, the general review for the uninitiated is interesting and stimulating, and in the field of surgery of the adrenal gland the author finds himself in a fascinating field of endocrinology with which he is very familiar and to which, together with his colleagues, he has made notable contributions.

\section{ESSENTIALS OF SYPHILOLOGY}

By R. H. Kampmeier. Blackwell, Oxford. r944. Price $25 \mathrm{~s}$.

This excellent little monograph of some 500 pages is written for the general practitioner and student. As stated in the preface it is not meant to be a treatise, and within its self-imposed limitations it succeeds admirably in presenting syphilis as it is met with in general practice.

The lay-out is good, the print is clear, the index is adequate and it contains 87 useful diagrams and photographs. The latter should be of real assistance to those who have a nodding acquaintance of the manifestations of the disease.

Little adverse criticism can be made of this book since it gives a sound account of the disease and its treatment, generally illustrated by a précis of case reports. relative to the point under discussion. This helpfue: method of presentation is much to be commended.

The chapter on serological aids to diagnosis is extremely? well done, and gives a well balanced account of the value of serum tests from the point of view of the clinician. $\frac{\bar{\omega}}{\partial}$

The book is suitably rounded off by short chapter\$ dealing with marriage and syphilis, control of syphilisu and educational measures relating to patients and public.

In short this book can be thoroughly recommended to all those practitioners who have devoted some of their $\overrightarrow{-}$ time to the practical study of syphilology and requires a textbook to brush up their knowledge and co-ordinatषo their ideas.

\section{HUMAN CONSTITUTION IN CLINICAL MEDICINE}

By George Draper, M.D., C. W. Dupertuis, Ph.D.

J. L. Caughey, M.D. Hamish Hamilton Ltd., London $\vec{D}$ for Paul B. Hober, Inc. New York, 1944. Price 21s. Judging by the more recent surgical and medica publications, there is obviously a strong and growing trend of medical opinion to revert more and more to biological principles for the explanation and correlation of clinical phenomena. Such a tendency is most welcome, for it shows that clinical observation, the very essence 850 medical science, is alive, stirring and bent on enlargif its outlook. It is a salutary antidote to the present afid popular conception that diagnosis is largely a matter $\$$ laboratory test tube or other ancillary form of investigation. This is an important omen, for it purposes tō study man as a whole as much as the particular disease? within him, and conforms to the teaching of Osler to® " Look at life as a whole: study its manifestations in order $\overrightarrow{\bar{O}}$ and disorder, in health and disease." By integrating the 3 form and functions of the several systems, a wider survey of disease and reaction and the personality of the individuato is obtained. Such a study broadens our knowledge on the part the subconscious animal reactions play in the emotions and instinctive behaviour of man.

With these preliminary remarks, the clinician will gather from this work by a team of Physicians of the Presbyterian Hospital, New York, something of the scope 3 of this well-written and documented book.

Its underlying principle is the study, from the medical? point of view, of the individual as a biological unit in relation to the pressure of his environment upon his organs, systems and personality.

The first chapters give useful hints to the clinician for developing his own clinical sense, and adapting it to new methods of investigation. Perhaps the most inter- $\omega$ esting of these is Somotyping, which takes into accoun such bodily characters as roundness, muscularity ando linearity. Another method includes Anthropometry $\frac{\Gamma}{\Phi}$ Such methods are correlated with clinical observations,? physiological and endocrine reactions, genetic control, growth and development, and the mosaic of andrology, 